\title{
Conceptual Parent
}

National Cancer Institute

\section{Source}

National Cancer Institute. Conceptual Parent. NCI Thesaurus. Code C80013.

Something from which one or more similar and separate things have developed, or to which they are attached. 